\title{
PADRÕES E ESTADOS COMPORTAMENTAIS DE RECÉM-NASCIDOS DURANTE O BANHO EM MATERNIDADE: POSSIBILIDADES DE REGULAÇÃO E TROCAS SOCIAIS
}

\section{NEWBORNS’ PATTERNS AND BEHAVIORAL STATES DURING THE BATH AT MATERNITY HOSPITAL: POSSIBILITIES OF SOCIAL REGULATION AND EXCHANGES}

GASPARETTO, S.; BUSSAB, V.S.R. Padrões e estados comportamentais de recém-nascidos durante o banho em maternidade: possibilidades de regulação e trocas sociais. Rev. Bras. Cresc. Desenv. Sumé, São Paulo, 10(1), 41-48, 2000.

\begin{abstract}
Resumo: Há um interesse cada vez maior em se compreender o recém-nascido e avaliar a adequação dos procedimentos de cuidado na maternidade. O objetivo do presente trabalho foi estudar as reações de bebês recém-nascidos (R.N.) durante o banho, dado pela atendente nas primeiras horas de vida e pela mãe dado após 72 horas de vida do bebê. 32 R.N., a termo, foram filmados na primeira situação e 20 destes, foram também filmados na segunda situação. Foram observados os estados e os comportamentos do R.N., como também os comportamentos do adulto. Os dados foram analisados de acordo com o sexo, tipo de parto, e situação (atendente/ primeiro banho x mãe/ segundo banho). Na primeira situação constatou-se que, nos casos de parto com intervenção, a frequência do estado de sonolência foi baixa antes e depois do banho, indicando transição brusca de estados calmos para agitação. Nenhuma diferença foi constatada durante o banho, o que sugere um procedimento abrupto, que iguala todos os bebés em estado de agitação. Na comparação das duas situações, os bebes mostraram mais agitação no banho dado pela atendente e mais alerta tranquilo no banho dado pela mãe, aparentemente em decorrência do tipo de estimulação promovida. O momento do banho é propicio para interação social, na medida em que se pode promover o alerta tranquilo, aumentando assim o seu contato com o meio.
\end{abstract}

Palavras-chave: comportamento neonatal; interação mãe-bebê; responsividade do adulto; cuidados em maternidade.

Há um interesse cada vez maior em se compreender o recém-nascido (R.N.) e avaliar a adequação dos procedimentos de cuidado na maternidade, na medida em que se tem demonstrado níveis de complexidade nas reações comportamentais de neonatos e dada a crescente evidência da importância das experiências iniciais no desenvolvimento.

As reações do bebê recém-nascido a estímulos externos e/ou internos podem ser observadas através de mudanças nos estados comportamentais dos bebês. Estes estados refletem a organização interna do bebê e a habilidade que ele tem para controlar os estímulos externos. BRAZELTON
(1973) sistematizou a Escala Neonatal de Avaliacão Comportamental, e definiu seis estados comportamentais, a saber: (1) sono profundo; (2) sono ativo; (3) sonolência; (4) alerta tranqüilo; (5) despertar ativo; e (6) choro intenso; considerando-os como um dos principais tópicos do exame comportamental e como uma matriz para a compreensão das reações dos bebês.

Quando são utilizados estímulos atrativos, auditivos ou visuais, o R.N. tem capacidade de atenção e alerta, e de suprimir respostas reflexas interferentes, de modo a responder com comportamentos muito previsíveis. Assim, ele responde e interage com seu meio ambiente desde o nasci-

1 Universidade de São Paulo - instituto de Psicologia - Departamento de Psicologia Experimental. Pesquisa financiada pelo CNPq. 
mento. A maneira pela qual o bebê responde aos estímulos apropriados ou não apropriados demonstra a complexidade de um SNC intacto e adaptável (BRAZELTON, 1984).

Várias pesquisas realizadas confirmam que o estado comportamental do bebê exerce uma influência fundamental na interação mãe-bebê (OSOFSKY \& DANZGER, 1974). Segundo THOMAN (1975), o estado age como um mediador das reações do bebê e eliciador das respostas do adulto em qualquer interação que ocorra entre a criança e o adulto. A habilidade do bebê para modificar seu alerta conforme as circunstâncias contribui para torná-lo parceiro na interação social.

Há um interesse especial na análise da atuação do adulto junto ao R.N., pois esta influencia a possibilidade de o bebê manter-se num estado motivacional apropriado, assim como pode influenciar seu desenvolvimento subseqüente.

Desde as constatações pioneiras de BOWLBY (1969) sobre o desenvolvimento do apego, tem se consolidado a verificação de que o vínculo mãe-bebê emerge da interação ajustada ao longo dos primeiros meses. Mãe e bebê necessitam de uma ligação forte para que se possa estabelecer os padrões de confiança indispensáveis ao desenvolvimento físico e emocional da criança (GOMES PEDRO, 1985).

Poderíamos dizer que o bebê tem padrões de comportamentos regulares e cíclicos aos quais o adulto ajusta o seu próprio comportamento, surgindo daí um diálogo que serve de base para o início da comunicação, à medida que se processa a interação entre a díade mãe-bebê. Esta pré-disposição social do R.N. facilita o caráter processual do desenvolvimento, em contínuo processo de ajustamento e adaptação à experiência (BRANCO, 1987).

Dados deste tipo levantam a questão de como os procedimentos empregados nas maternidades interferem na interação mãe-bebê. Convém ainda considerar que há também interesse em se avaliar a própria interação dos demais adultos com o R.N. na maternidade, lembrando-se a potencial sensibilidade do período para o bebê.

A importância da mãe depende da sua consistência de afeto. À medida em que o bebê se desenvolve em termos de consciência social e de mobilidade, um código consistente de sinalização emocional guia a formação de novas relações e o ajustamento de vínculos com irmãos e outras pessoas. As evidências disponíveis indicam que a função básica das emoções humanas é regular as representações mentais de contatos e relações interpessoais (TREVARTHEN, 1984).

Muitas pesquisas sugerem-nos que o contato inicial mãe-bebê é de especial importância para o desenvolvimento posterior da criança, como também para uma boa formação do vínculo (KLAUS \& KENNEL, 1989). Desta forma, resolvemos estudar uma das situações de primeiros cuidados, em maternidade, após o nascimento do bebê.

O objetivo do presente trabalho foi investigar as reações dos bebês recém-nascidos, em um ambiente hospitalar, durante a situação de banho, comparando as suas reações ao banho dado pela atendente e, subseqüentemente, no banho dado pela mãe. Analisou-se também como o adulto respondia aos sinais do bebê, com especial atenção a possíveis reações dos adultos às mudanças de estados comportamentais do R.N. e às possíveis tentativas do adulto de conduzir os bebês a estados de calma ou alerta. A situação de banho caracteriza-se por um alto nível de manipulação do bebê. Estas manipulações podem produzir diversas reações no recém-nascido. Esta é uma situação potencialmente reveladora das características da reação do R.N. aos tipos de manipulações e da adequação do procedimento. Trata-se, portanto, de uma situação que propicia uma série de trocas e ajustes interacionais entre o adulto e a criança.

\section{MÉTODO}

\section{Sujeitos}

Foram observados trinta e dois bebês recém-nascidos, dezesseis meninas e dezesseis meninos, no primeiro dia - com até duas horas após o parto - e terceiro dia de vida - com até setenta e duas horas depois do nascimento - em um hospital da cidade de São Paulo - Brasil. Dos R.N. estudados, treze nasceram de parto fórceps, dez de parto normal e nove de cesárea. Foi administrada anestesia em vinte e oito mães e as quatro restantes não tomaram este tipo de medicamento. A idade das mães variou de dezasseis a quarenta e um anos: destas, doze eram primíparas e vinte já tinham tido mais de uma gestação. A idade gestacional dos bebês, pelo método de capurro somático, variou de 36 5/7 a 41 2/7 semanas de gestação. O peso dos R.N. ao nascer variou de 2800 gramas a 3900 gramas. O Apgar de primeiro minuto variou de 6 a 9 e do quinto minuto de 8 a 10. Dos trinta e dois sujeitos observados, quinze eram de cor parda, quatorze de cor branca e três de cor preta. Todos os sujeitos eram de classe social baixa.

\section{Procedimento}

O estudo foi realizado em um hospital universitário. Trinta e dois R.N. foram observados no primeiro banho com a atendente e destes, vin- 
te também foram observados com a mãe no segundo banho. A Primeira sessão de observação foi feita nas duas primeiras horas de vida do R.N. durante a situação de banho realizada pela atendente, na sala de observação I do berçário. A segunda sessão de observação foi realizada no alojamento conjunto, na maternidade, em situação de banho realizada pela mãe, depois de setenta e duas horas do nascimento da criança (antes da alta). Todas as mães foram preparadas pelas enfermeiras com aulas de como dar o banho em seus bebês.

Com a permissão das mães e do hospital, foram realizadas gravações em vídeo. Todos os sujeitos foram filmados por periodos de vinte minutos, procurando cobrir situações imediatamente anteriores ao banho, o banho todo, e os minutos que se seguiram até completar vinte minutos de sessão. O registro dos comportamentos foi feito de modo cursivo, em periodos de quinze segundos. Ou seja, o video foi paralisado a cada intervalo, de modo que as transcrições fossem feitas.

Foram registrados os Estados Comportamentais descritas por BRAZELTON (1973) em seu teste NBAS: - Estado 1 (sonoprofundo); Estado 2 (sono REM); - Estado 3 (sonolência); Estado 4 (alerta inativo); - Estado 5 (alerta ativo); - Estado 6 (choro intenso). Alguns dos itens registrados nas sessões de observação referentes aos comportamentos do R.N., foram baseados na definição de ALS (1984). Os comportamentos foram:

I. Comportamentos indicativos de instabilidade autonômica: - Tremor: O R.N. treme uma parte ou todo o corpo, por exemplo, tremor dos braços e pernas e/ou corpo todo.

II. Comportamentos Auto-Tranqüilizadores: - Mão-Boca: O R.N. traz sua mão ou dedos para sua boca, mas não chega sugar; Procura-sugar: O R.N. estende seus lábios ou abre sua boca na procura da mão ou dedos para sugar, geralmente movendo sua cabeça enquanto o faz; - Sugar: O R.N. suga seus próprios dedos ou mão.

III. Comportamentos que indicam mal-estar (irritabilidade): - Movimento Boca: O R.N. faz movimentos com a boca com os lábios /ou maxilar, que sugerem expressão de choro. - Choramingo: O choro propriamente dito foi computado na análise dos estados, correspondente ao estado 6. Espirro: O R.N. expele o ar forcosamente pela boca e nariz em uma explosiva e espasmódica ação involuntária.

IV. Comportamento de Alerta: - Olhar. O R.N. olha para face do adulto e/ou objetos atrativos do meio. O R.N. deve apresentar olhos abertos, com olhar brilhante, focalizando a atenção sobre as fontes de estímulos. A atividade motora é mínima (BRAZELTON, 1973).

O registro dos comportamentos do adulto (mãe e atendente), foram baseados na definição de GOMES PEDRO (1985). Os comportamentos foram:

I. Comportamento Afetuoso: - Beijar: qualquer tipo de contato da boca da mãe e/ou atendente com o R.N. (incluindo contato face a face); -. Sorrir: sorri olhando para o R.N. e/ou outras pessoas; - Face/Face: A mãe e/ou atendente ergue o R.N. em posição vertical de forma que seus olhos encontrem diretamente a face do R.N. (o R.N. pode estar ou não olhando para face do adulto); . Acariciar: contato afetuoso da mão ou dedos em movimento no corpo do R.N.; -. Tocar: contato da mão ou dedos com o corpo do R.N., sem movimento da mão ou dedos.

II. Comportamentos de Atenção: - Falar para outra pessoa: Falar para alguém presente ou próximo, incluindo o observador; - Olhar para outra coisa ou pessoa: a mãe e/ou atendente desvia o seu olhar do R.N.; - Falar para o R.N.: A mãe e/ou atendente fala para o R.N.

\section{RESULTADOS}

\section{Análise da primeira sessão de observação}

Os dados referentes aos 32 sujeitos, na primeira sessão de observação, foram analisados através de Análise de Variância (ANOVA), um delineamento fatorial 2 (sexo: masculino e feminino) x 3 (tipo de parto: normal, fórceps, cesárea) sendo analisadas: 1) a predominância dos estados comportamentais em cada fase da sessão (antes, durante e depois do banho) e 2) as freqüências dos comportamentos em geral.

\section{a) Estados comportamentais}

Na fase antes do banho, não foram constatados efeitos significativos de sexo sobre os estados. Em função do tipo de parto, verificou-se uma tendência de efeito quanto à predominância do estado 3 - sonolência $[\mathrm{F}(2,24)=3.110$; $\mathrm{p}<\mathrm{O}$. $10]$, nada sendo constatado para os demais estados. O teste de Tuckey demonstrou diferenças significativas entre os tipos de parto para o estado 3: nos bebês de parto normal ( $\mathrm{M}=32,1 \%$ 11,2\%) este estado é mais frequente do que nos demais; além disso, os de fórceps ( $M=16,1 \% \pm 9 \%)$ também superaram os de cesárea $(\mathrm{M}=\overline{0} \% \pm 0 \%)$ ( $\mathrm{p}<005)$.

Durante o banho constatamos que não houve resultados estatisticamente significativos para nenhum dos estados comportamentais $(1,2,3,4$, 5 e 6), analisados em trinta e dois sujeitos durante 
o banho dado pela atendente, em função de sexo, tipo de parto ou interação sexo (A) x parto (B).

Na fase depois do banho, o estado comportamental 3 variou significativamente em função do tipo de parto $[\mathrm{F}(2,26)=3.507, \mathrm{p}<0.05]$. O teste "post-hoc” de Tuckey revelou que os bebés de parto normal $(\mathrm{M}=46,2 \% \pm 10,5 \%)$ ficaram mais em estado 3 que os de parto cesárea $(\mathrm{M}=21,3 \% \pm 10,9 \%)$ e que os de parto fórceps $(\mathrm{M}=13,6 \% \pm 6,0 \%)(\mathrm{p}<0.05)$. A Figura 1 - indica a média em porcentagem do estado comportamental 3 depois do banho para meninos e meninas nos diferentes tipos de parto, podendo-se notar a predominância deste estado no caso de parto normal.

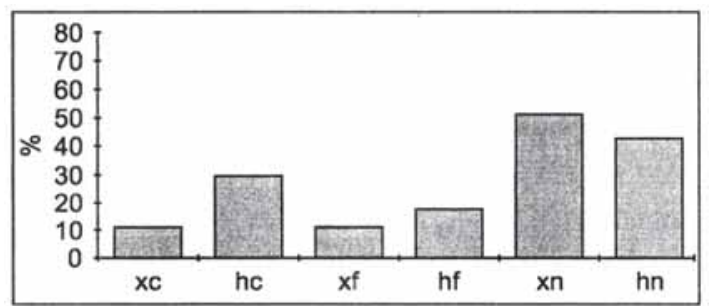

Figura 1: Média do estado comportamental 3 depois do banho para meninos (h) e meninas (x) nos diferentes tipos de parto: cesárea (c); fórceps (f); normal (n).

\section{b) Comportamento do R.N.}

A análise das freqüências dos comportamentos em função de sexo e tipo de parto foi feita considerando-se a sessão como um todo, dada a baixa freqüência de algumas categorias.

O comportamento procura-sugar variou significativamente em função do tipo de parto $[\mathrm{F}(2.26)=4.539, \mathrm{p}<0.05]$. Esteve mais presente nos bebês nascidos de parto cesárea $(M=4,6 \pm 19)$ do que nos nascidos de fórceps $(\mathrm{M}=0,9 \pm 0,3) \mathrm{e}$ normal $(\mathrm{M}=0,6 \pm 0,2)(\mathrm{p}<0.05)$. Não houve diferença em função de sexo.

Para os demais comportamentos analisados como o Sugar; Olhar; Choramingo; Espirro; Tremor, Mão-Boca e Movimento-Boca não encontramos nenhum resultado significativo em função dos fatores sexo (A), parto (B) ou interação sexo (A) x parto (B).

\section{Análise em conjunto das duas sessões de observação}

Os dados referentes aos 20 bebês para os quais realizamos duas sessões de observação foram analisados através de uma Análise de Variância (ANOVA) com delineamento 2 (Sexo: feminino x masculino) X 3 (Tipo de parto: cesárea x fórceps x normal) X 2 (Adulto: $1^{\circ}$ banho (atendente) x $2^{\circ}$ banho (mãe). Foi utilizado o tratamento "Mixed ANOVA" para dois fatores independentes (sexo e tipo de parto) e um fator dependente, ou seja, com medidas repetidas $\left(1^{\circ}\right.$ banho/ $2^{\circ}$ banho).

\section{a) Estados comportamentais}

Durante o banho os R.N. permaneceram mais no estado 5 - alerta agitado- quando banhados pelas atendentes $(\mathrm{M}=53,6 \% \pm 5,1 \%)$ do que quando banhados pelas mães $(\mathrm{M}=34,2 \% \pm 5,1)$ $[\mathrm{F}(1,14)=9.052, \mathrm{p}<0.01]$. A Figura 2 ilustra a média do estado comportamental 5 exibida por meninos e meninas de diferentes tipos de parto nas duas situações de banho. Pode-se observar que em bloco, as freqüências do estado 5 associados às mães (m) são mais baixas.

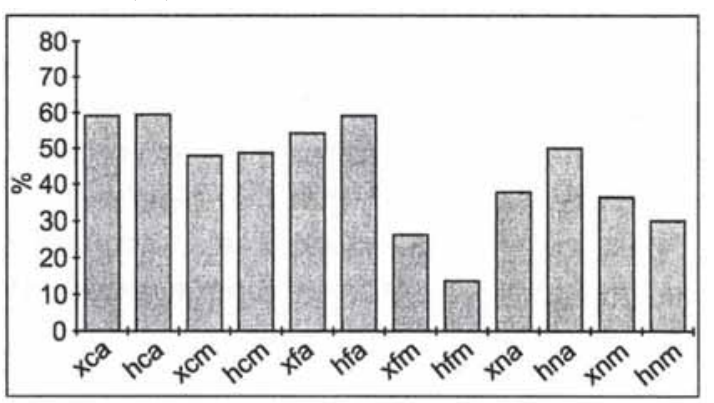

Figura 2: Média do estado comportamental 5 exibida por meninos (h) e meninas (x) de diferentes tipos de parto: cesárea (c); fórceps (f) e normal (n); nas diferentes situações de banho, com a atendente (a) e com a mãe (m).

Por sua vez, os bebês quando banhados pelas mães ( $\mathrm{M}=18,1 \% \pm 4,6 \%)$ ficaram mais em alerta tranqüilo do que quando banhados pelas atendentes $(\mathrm{M}=3,1 \% \pm 1,7 \%)[\mathrm{F}(1,14)=8.263$, $\mathrm{p}<0.05]$. A Figura 3 ilustra a média para meninos e meninas nos diferentes tipos de parto com a atendente e com a mãe. Neste caso a Figura mostra que os blocos correspondentes às mães são mais altos do que os referentes às atendentes.

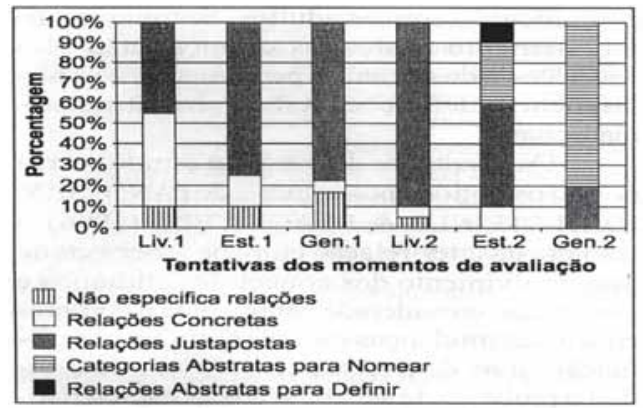

Figura 3: Média do estado comportamental 4 exibidapormeninos (h) e meninas (x), nos diferentes tipos de parto: cesárea (c), fórceps (f) e normal (n). 
Depois do banho, os R.N. permaneceram mais em alerta com as mães $(M=30,3 \% \pm 9,3 \%)$ do que com as atendentes ( $\mathrm{M}=2,6 \% \pm 1,2 \%)$ $[\mathrm{F}(1,10)=11.937, \mathrm{p}<0.01]$. Foi observada também uma significância no efeito principal sexo $[\mathrm{F}(1,10)=$ ó.748,p $<0.05]$. Os meninos $(\mathrm{M}=26,9 \%$ $\pm 8,1 \%$ ) ficaram mais em estado 4 do que as meninas ( $M=6 \% \pm 5,7 \%)$. A Figura 4 mostra a média do estado comportamental 4 para meninos e meninas nos diferentes tipos de parto com a atendente e a mãe.

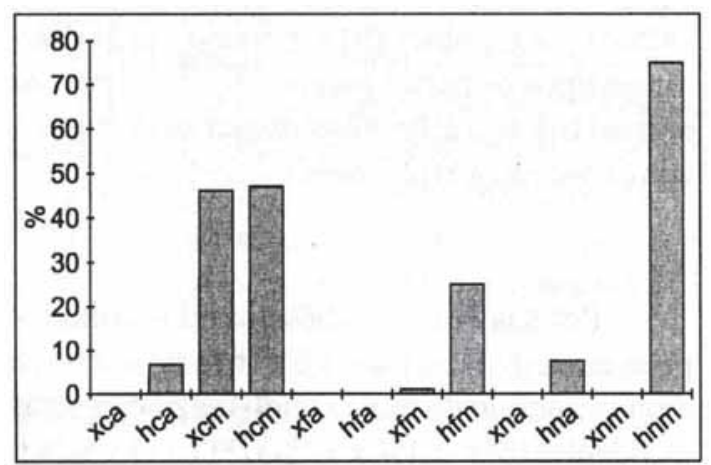

Figura 4: Média do estado comportamental 4 apresentado por meninos (h) e meninas (x) nos diferentes tipos de parto: cesárea (c); fórceps (f); normal (n). Na situação de observação com a atendente (a) e com a mãe (m).

Houve uma interação significativa entre sexo e adulto na variável do estado 6 - choro intenso $-[\mathrm{F}(1,10)=5.527, \mathrm{p}<0.05]$. Os meninos $(\mathrm{M}=17,3 \% \pm 7,3 \%)$ choraram mais do que as meninas $(\mathrm{M}=14,7 \% \pm 6,5)$ na primeira sessão de observação ( $1^{\circ}$ banho-atendente) $(\mathrm{M}=18,8 \% \pm$ $6,6 \%)$ do que na segunda sessão ( $2^{\circ}$ banho-mãe) $(\mathrm{M}=13,1 \% \pm 7,2 \%)$.

Percebemos que as meninas $(\mathrm{M}=33,2 \% \pm$ $10,6 \%$ ) ficaram mais em estado 3 depois do banho do que os meninos ( $\mathrm{M}=10,8 \% \pm 5,4 /)$. $\mathrm{O}$ efeito principal sexo foi significativo $[\mathrm{F}(1,10)=$ 5.823, $\mathrm{p}<0.05$ ]

\section{b) Comportamento do $\mathrm{RN}$.}

Os bebês ficaram mais em alerta com as mães $(\mathrm{M}=14,3 \pm 3,5)$ do que com as atendentes $(\mathrm{M}=1,4$ \pm 0,6) $[\mathrm{F}(1,14)=12.885, \mathrm{p}<0.01]$. Houveumatendênciano efeito principal sexo $[\mathrm{F}(1,14)$ $=3.109, \mathrm{p}<0.10]$. Os meninos $(\mathrm{M}=11,2 \pm 3,6)$ apresentaram maior tendência para o comportamento de olhar que as meninas $(\mathrm{M}=4,5+1,6)$.

Para o comportamento do RN. Movimento/ Bocaencontramos significanciano efeito principal adulto $[F(1,14)=9.924, p<0.01]$. Os sujeitos apresentaram mais este comportamento na primeira sessão de observação $(M=3,9 \pm 1,2)$ do que na segunda sessão de observação $(\mathrm{M}=0,1 \pm 0)$. Os recém-nascidos procuraram-sugar mais nas primeiras horas de vida $(\mathrm{M}=2,7 \pm 0,9)$ do que com setenta e duas horas $(\mathrm{M}=0,8 \pm 0,4)[\mathrm{F}(1.14)=4.497$, $\mathrm{p}<0.05]$. Também houve significância no efeito principal parto para o comportamento procura-sugar $[\mathrm{F}(2,14)=4.250, \mathrm{p}<0.05]$. O teste "Post-hoc" $(p<0.05)$ confirmou que os bebes nascidos de parto cesárea $(\mathrm{M}=3,7 \pm 1,3)$ tiveram mais o comportamento procura-sugar do que os nascidos de parto fórceps $(M=0,710,3)$ e normal $(M=0,510,2)$.

A análise para o comportamento choramingo revelou significância para o efeito principal parto $[\mathrm{F}(2,14)=5.887, \mathrm{p}<0.05]$. O teste Tuckey $(p<0.05)$ mostrou que os R.N. nascidos de parto cesárea $(\mathrm{M}=6,2 \pm 1,6)$ choramingam mais que os bebês nascidos de parto fórceps ( $\mathrm{M}=2,5 \pm$ $0,7)$ e parto normal $(\mathrm{M}=1,3 \pm 0,7)$

\section{c) Comportamento do adulto}

Observamos que as mães ( $\mathrm{M}=4,9 \pm 0,7)$ apresentaram mais o comportamento afetuoso do que as atendentes $(\mathrm{M}=1,1 \sim 0,3)[\mathrm{F}(1,14)=$ 25.257 , p $<0.01]$. Encontramos também uma tendência a significância do fator principal sexo $[\mathrm{F}(1$, $14)=4.325, \mathrm{p}<0.10$ ]. Houve mais comportamento afetuoso com os meninos $(\mathrm{M}=3,7 \pm 0,7)$ do que com as meninas ( $\mathrm{M}=2,2 \pm 0,5)$. As mães $(\mathrm{M}=1,2 \pm 0,3)$ apresentaram mais o comportamento Olhar coisas e/ou pessoas do que as atendestes $(M=0,5+0,1)$. A tendência encontrada foi no efeito principal adulto $[F(1,14)=4.287$, $\mathrm{p}<0.10]$. O comportamento Falar Pessoas foi mais freqüente nas mães $(M=6 \pm 1,6)$ do que nas atendentes $(\mathrm{M}=3,4 \pm 0,7) \overline{[F}(1,14)=3.658$, $\mathrm{p}<0.10]$. Não encontramos resultados significativos para o comportamento Falar/R.N. em nenhum dos fatores.

\section{DISCUSSÃO E CONCLUSÃO}

Os resultados revelaram que o estado comportamental 3 (sonolência) é mais freqüente nos bebês nascidos de parto normal, do que nos outros tipos de parto, nas situações antes e depois do banho. A baixa freqüência deste estado nos bebês que tiveram parto com intervenção parece sugerir que, nestes, a passagem de estados calmos para os estados de agitação é mais abrupta, uma vez que o estado 3 é intermediário. Sugere também maior concentração, nestes casos, nos estados 5 e 6, uma vez que o estado 4 é baixo. Ainda nesta linha, chama a atenção a ausência total do estado 3 nos bebês de cesárea, antes do banho, e uma presença mais forte de choro nos bebês de fórceps, depois do banho. 
O choro é um indicador de perturbação e funciona no sentido de reduzi-la. O choro tem muitas finalidades no recém-nascido, das quais as mais importantes são as de afastar estímulos dolorosos ou perturbadores. Parece também ter importância para reduzir distúrbios no sistema nervoso central (BRAZELTON, 1984). A presença mais forte de choro nos bebês de fórceps depois do banho indica certa permanência da perturbação nestas crianças depois deste tipo de manipulação.

A ausência de diferença de distribuição de estados em função do tipo de parto durante o primeiro banho parece indicar que os procedimentos do banho levam a uma agitação de todos os bebês (como veremos adiante), suplantando outras diferenças perceptíveis em interações menos intensa, ou seja, independentemente do tipo de parto, todos os bebês tendem a se agitar durante o banho.

O conhecimento do estado comportamental do bebê poderia ajudar o adulto no ajustamento das ocasiões de intervir e cuidar. A quantidade correta de estimulação fornecida deveria ser baseada nos indicadores de retorno do comportamento emocional do bebê. Os relatos dos pais e as revisões sobre cuidados com crianças não apresentam evidências de um uso consciente destes indicadores pelos adultos (PAPOUSEK \& PAPOUSEK, 1984), embora mostrem este uso efetivo em condições favoráveis.

Outro indicador diferencial, em função do tipo de parto, provém de uma única diferença encontrada com relação ao comportamento do recém-nascido: freqüência mais alta de procurar sugar em crianças de cesárea, significativa de auto-apaziguamento.

Pode-se dizer que não encontramos nesta análise diferenças em função do sexo: o único resultado neste sentido foi o de uma tendência para mais comportamento de mão-boca em meninos. A coordenação mão-boca aparece com poucas horas após o nascimento do bebê (ROCHAT, BLASS \& HOFFNEYER, 1988).

GOMES PEDRO (1985) relata que os bebês que tiveram contato precoce com suas mães, no primeiro dia de vida, obtiveram melhores resultados no comportamento mão-boca. Estes resultados indicam uma melhor coordenação motora com uma auto-organização mais eficiente nos mecanismos de controle dos recém-nascidos, especialmente em situação de maior "stress"; uma vez que estes padrões parecem ter uma função auto-apaziquadora.

Foram constatadas duas diferenças significativas na distribuição dos estados, entre as duas situações de banho. Os bebês permaneceram mais em agitação (estado 5), durante o primeiro banho do que durante o segundo e mais em alerta (estado 4) neste do que no primeiro.

Cabe a discussão, neste ponto, do efeito do fator adulto (atendente x mãe) ou idade (primeiro dia x terceiro dia) na produção destes resultados. Além disso, ambos fatores poderiam estar atuando. Embora o fator idade possa estar atuando, temos motivos para supor que o tipo de manipulação possa ser um dos responsáveis por essas variações. Constatamos que os adultos que manipularam o bebê estavam mais voltados para cumprir uma rotina de higiene, com etapas bem definidas, do que para reagir aos sinais de perturbações do bebê: isto era claro no caso da atendente e de certa forma era exigido da mãe, na medida em que esta foi treinada para etapas do banho. Como já mencionamos, durante o banho, os recém-nascidos ficaram mais entre os estados 5 (agitação) e 6 (choro profundo). Desta forma, poderíamos dizer que o adulto que banhava o bebê estava mais preocupado em completar e realizar da forma carreta as etapas do banho do que acalmar o bebê, perdendo assim a oportunidade de interação e observação dos comportamentos do bebê.

Seria útil orientar a interação dos adultos no sentido de aumentar sua sensibilidade às alterações de estados dos bebês. O “estado” torna-se uma matriz para compreensão de suas reacões. Ele qualifica o estímulo como apropriado ou inapropriado para a organização da criança. Para quase todos os níveis de maturação, o comportamento produzido por estímulos apropriados em “estados” apropriados demostrará a complexidade de um SNC intacto e adaptável (BRAZELTON, 1984).

Constatamos também diferenças significativas na freqüência do estado 4, alerta, depois do banho, no mesmo sentido já citado, ou seja, este estado é mais comum depois do banho com a mãe. Verificamos também um efeito de sexo: os meninos ficaram mais em alerta que as meninas.

A mãe influencia profundamente a regulação dos estados de vigilância do bebê. Se um R.N. oscilar sem transição entre o sono e os gritos, uma mãe particularmente paciente e sensível aos menores sinais enviados por seu filho poderá progressivamente levar o bebê a um estado calmo e atento (MAZET \& STOLURE, 1990).

Análises preliminares dos videos sugerem que as mães tendem a estimular mais os meninos. Um estudo de GOTTFRIED, SEAY \& WISMAR (1987) mostra-nos que as mães que tiveram o primeiro filho do sexo masculino quando comparadas a mães de segundo filho do sexo feminino despenderam mais comportamento de pré-vínculo. Os resultados também sugeriram que as mães estabeleceram mais contato corporal afetuoso com 
os meninos quando estes eram o primeiro filho. Contudo, esta é uma hipótese de diferença de tratamento materno em função do sexo do bebê que precisará ser mais bem investigada em estudos posteriores.

Em contraste, as meninas tenderam a ficar mais em estado 3 - sonolência - depois do banho com as mães, do que os meninos. Os dados referentes a comportamento são compatíveis aos resultados obtidos na análise dos estados.

Um dos indicadores de auto-apaziguamento, procura sugar, ocorreu mais na primeira situação de observação do que na segunda. O padrão mão-boca, na primeira situação, foi mais freqüente nos meninos do que nas meninas. O comportamento mão-boca também é um indicador de autoapaziguamento ou tranqüilizador (ROCHAT, BLASS \& STOLURE, 1990).

As mães apresentaram mais o comportamento afetuoso do que as atendentes. É possível que este padrão afetuoso esteja relacionado com a maior permanência dos bebês em alerta (estado 4). Houve também uma tendência maior para o comportamento afetuoso com os meninos do que com as meninas. Nossos resultados se alinham aos de GOMES PEDRO (1985). Seus resultados mostraram que as mães dos meninos do grupo experimental foram mais afetuosas, enquanto que as mães das meninas eram mais distraídas relativamente ao grupo controle.

É dificil estabelecer o sentido desta correlação de comportamento afetuoso em função do sexo. Lembrando-se que os meninos ficam mais em alerta do que as meninas, na situação com a mãe, durante e depois do banho e que as meninas ficam mais sonolentas depois do banho, não é possível decidir se estes padrões são decorrentes de maior estimulação das mães sobre os meninos ou se de maior sinalização receptiva dos meninos nesta interação.

Poderíamos dizer então que o banho tem uma importância fundamental para organização do bebê, pois a estimulação tátil é feita neste momento. Além de que, o momento do banho é propício para interação social na medida em que pode promover o alerta tranqüilo, aumentando assim o seu contato com o meio.

A situação de banho serve também para manter um contato recíproco mãe-bebê. Verificamos isto quando o R.N. ficou em alerta tranqüilo durante o banho. Quando falamos de estimulação e interação, estamos, evidentemente falando do afeto.

Os primeiros contatos da mãe com seu bebê são de fundamental importância para a díade e a qualidade destes primeiros contatos pode influenciar o estabelecimento e desenvolvimento do apego. É claro que perturbações nestes primeiros contatos podem ser superadas. Entretanto, facilitações nestes primeiros contatos aumentam as possibilidades de um desenvolvimento mais harmonioso.

Ao estudarmos o bebê recém-nascido, nos três primeiros dias de vida, em situação natural, pretendíamos entre outras coisas mostrar a importância do conhecimento do adulto das reações e estados comportamentais do R.N. Muitos comportamentos do adulto dirigidos ao bebê, em uma situação de interação são inconscientes. Parecenos necessário que se introduza nas rotinas hospitalares de maternidade instruções sobre o comportamento e reações do bebê.

A equipe de saúde materno-infantil deverá ter uma participação importante no esclarecimento e reforço no que diz respeito ao relacionamento adequado da mãe com seu filho. Desta forma, a equipe multidisciplinar faria um trabalho preventivo, no sentido de propiciar um desenvolvimento sádio para a criança, uma vez que os primeiros contatos são muito importantes para o ajustamento inicial da díade mãe-bebê e facilitadores do processo de formação de apego. Outro ganho potencial seria a redução de eventos perturbadores na manipulação do bebê por parte da equipe.

A aplicação dos conhecimentos sobre a importância das experiências iniciais neonatais no desenvolvimento do bebê tem sido feita de maneira fragmentada e parcial. Mudanças de procedimentos, tais como o estabelecimento de alojamento conjunto, embora muito favoráveis, nem sempre garantem mudanças efetivas nas experiências produzidas. Pesquisas de campo sistemáticas, associadas a cursos de formação e de treinamento, parecem necessários, mesmo porque é preciso que ocorra uma modificação básica de atitude quanto aos fatores relevantes na situação de atendimento em maternidade (GASPARETTO \& BUSSAB, 1994).

Agradecimentos: Ao Hospital Universitário e todas as enfermeiras, atendentes e mães que tornaram possível a realização deste trabalho. 


\begin{abstract}
There is an increasing interest in understanding the newborn child and evaluating the adequacy of care procedures at the maternity hospital. The aim of this research was to study the newborns' reactions during the baths, respectively given by the nurse within the first hours of life, and by the mother, seventy-two hours after birth. Thirty-two newborns (full-term gestation) were filmed in the first situation and twenty of them were also filmed in the second one. The states'and behaviors of the newborns were investigated, as well as the adult behavior. Data were analyzed according to sex, type of birth, and situation (nurse x mother) (analysis of variance ANOVA). We performed an ANOVAS on the data of the first situation (nurse): in cases of birth with surgery the equency of semi-dozing state was lower, before $[F(2,24)=3.11, p<0.10]$ and after $[\mathrm{F}(2,24)=3.50, \mathrm{p}<0.05]$ the bath, suggesting an abrupt procedure that results in an agitation state which is similar for ali the babies. Then, we performed ANOVAS including the comparison of the two situations: the neonates showed more agitation in the bath givenby the nurse $[F(1,14)=9.05, p<0.01]$ and calmer alert inthe bath given by the mother $[F(1,14)=8.26$, $\mathrm{p}<0.05)$, probably a consequence ofthe kind of stimulation provided. The bath is seen as an adequate moment for social interaction as it can promote the calm alert state, thus increasing the contact between the newborn and the environment.
\end{abstract}

Key-words: neonatal behavior; mother-infant interaction; adult responsiveness; maternity care.

\section{REFERENCIAS BIBLIOGRÁFICAS}

ALS, H. Manual for the naturalistic observation of newborn ,behavior (preterm and fullterm infants). Revision. Boston, The Children's Hospital, l 981, 1984.

BOWLBY, J. Attachment and loss. attachament. New York, Basic Books, 1969.

BRANCO, A.V. A relação mãe-criança: Considerações sobre a ontogênese da "natureza”social do ser humano. Psicologia: Teorias e Pesquisa, 3(3): 296 300, 1987.

BRAZELTON, T.B. Neonatal Behavioral Assessment Scale. London, Willian Heinemann Medical Books, 1973.

BRAZELTON, T. B. Competência comportamental do recém-nascido. In: GORDON, B.A. (Ed.). Neonatologia, fsiopatologia e tratamento do recémnascido. Rio de Janeiro, Editora Médica e Científica LTDA, 1984. p.329-355.

GASPARETTO, S. ; BUSSAB, V. S. R. A necessidade de um trabalho preventivo em maternidade: instruções sobre o comportamento do recém-nascido. Revista Brasileira de Crescimento e Desenvolvimento Humano, 4(2): 3034,1994.

GOMES PEDRO, J.C.G.A. A relação mãe-f lho: influencia do contacto precoce no comportamento da díade. Lisboa, Imprensa Nacional Casa da Moeda, 1985.

GOTTFRIED,N.W.; SEAY, B.M.; WISMAR, K. Neonatal sex and birth-order effects on behaviors of mothers and temporary caretakers during bottlefeeding. Journal of Genetic Psychology, 14(4): 479-497,1987.
KLAUS, M.H.; KENNEL, J.H. Pais/bebe - a formação do apego. Porto Alegre, Ed. Artes Médicas, 1989.

MAZET, R; STOLURE, S. Manual de psicopatologia do recém-nascido. Trad. de F.F. Settineri. Porto Alegre, Artes Médicas, 1990.

OSOFSKY, J.D.; DANZGER, B. Relationships between neonatal characteristics and motherinfant interaction. Developmental Psychology, 1974; 10(1): 124-130.

PAPOUSEK, H.; PAPOUSEK, M. Learning and cognition in the every day life of human infants. Advances in the Study of Behavior. New York, AcademicPress,1984.Vol. 14: 127163.

ROCHAT, P.; BLASS, E.M.; HOFFNEYER, L.B. Oropharyngeal control of hand-mouth coordenation in newborn infants. Developmental Psychology, 24(4): 459-463, 1988.

THOMAN, E.B. Sleep and wake behavior in neonates: consistencies and consequences. Merril-Palmer Quartely, 21 (4): 295-314, 1975.

TREVARTHE^N, C. Emotions in infancy regulations of contact and relationship with persons. In: Scherer, K.r.; Ekman, P. (Eds.). Approaches to emotion. Londres, Lawrence Erlbaum, 1984. 